\title{
DÜBLIN
}

Technological University Dublin

ARROW@TU Dublin

\section{Analysis of Strain Transfer to FBG's for Sensorized Telerobotic End-effector Applications}

\author{
Dean Callaghan \\ Technological University Dublin, dean.callaghan@tudublin.ie \\ Mark McGrath \\ Technological University Dublin, mark.mcgrath@tudublin.ie \\ Ginu Rajan \\ Technological University Dublin, ginu.rajan@tudublin.ie
}

See next page for additional authors

Follow this and additional works at: https://arrow.tudublin.ie/engschmanbk

Part of the Biomedical Devices and Instrumentation Commons

\section{Recommended Citation}

Callaghan, D., McGrath, M., Rajan, G., Coyle, E., Semenova, Y., Farrell, G.: Analysis of Strain Transfer to FBG's for Sensorized Telerobotic End-Effector Applications. Advances in Robotic Research. Theory, Implementation, Application, Springer Verlag, Part 3, Pages 65-75, June 2009. DOI:10.21427/58th-2y98

This Book Chapter is brought to you for free and open access by the School of Manufacturing and Design Engineering at ARROW@TU Dublin. It has been accepted for inclusion in Books/Book chapters by an authorized administrator of ARROW@TU Dublin. For more information, please contact arrow.admin@tudublin.ie, aisling.coyne@tudublin.ie, gerard.connolly@tudublin.ie.

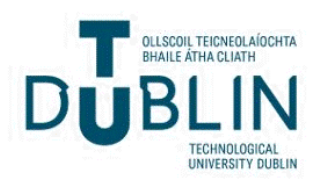




\section{Authors}

Dean Callaghan, Mark McGrath, Ginu Rajan, Eugene Coyle, Yuliya Semenova, and Gerald Farrell 


\title{
Analysis of Strain Transfer to FBG's for Sensorized Telerobotic End-Effector Applications
}

\author{
Dean J. Callaghan, Mark M. McGrath, Ginu Rajan, Eugene Coyle, Yuliya \\ Semenova and Gerald Farrell
}

Faculty of Engineering, Dublin Institute of Technology, Ireland.

\begin{abstract}
Sensorized instruments which cater for the measurement of interaction forces during surgical procedures are not available on current commercial Minimally Invasive Robotic Surgical (MIRS) systems. This paper investigates the effectiveness of advanced optical sensing technology (Fiber Bragg Grating) as surgical end effector strain/force sensors. The effects of adhesive bonding layer thickness and length are specifically addressed owing to their importance for effective strain transfer and ensuring compactness of the resulting sensing arrangement. The strain transfer characteristics of the compound sensing arrangement are evaluated by the examination of shear transfer through the fiber coating and adhesive layers. Detailed analysis of the sensing scheme is facilitated through the use of FEA. Validation of the resulting models is achieved through experimentation carried out on an application-specific evaluation platform. Results show that strain values from an FBG are comparable to that of an electrical strain gauge sensor.
\end{abstract}

\section{Introduction}

Current commercially available MIRS systems greatly augment the surgeon's ability to carry out an operating procedure effectively but lack the facility to relay haptic (kinesthetic and tactile) information to the user.

\footnotetext{
Dean Callaghan, School of Manufacturing \& Design Eng., DIT, e-mail: dean.callaghan@dit.ie

Mark McGrath, School of Manufacturing \& Design Eng, DIT, e-mail: mark.mcgrath@dit.ie

Ginu Rajan, Photonics Research Centre, DIT, e-mail: ginu.rajan@ dit.ie

Eugene Coyle, School of Electrical Engineering Systems, DIT e-mail: eugene.coyle@dit.ie

Yuliya Semenova, Photonics Research Centre, DIT, e-mail: yuliya.semenova@dit.ie

Gerald Farrell, School of Electronic \& Communications Eng., DIT, e-mail: gerald.farrell@ dit.ie
} 
This failure to provide for force measurement at the instrument end-effector restricts the effectiveness of MIRS systems in the detection of interaction, cutting and grasping forces during surgical tasks. A major obstacle in the provision of force feedback in MIRS systems is attributed to the actual measurement of the interaction forces [1].

Research is ongoing into the use of strain/force sensors for the measurement of interaction forces at the instrument-tissue interface. Resistive strain gauge technology has been utilized either in the form of a modular sensor [2, 3] or attached onto the instrument trocar [4-7]. These arrangements only measure interaction and bending forces on the trocar and do not measure grasping and cutting forces. One approach to overcoming this problem is the placement of strain/force sensing transducers either onto the instrument tip or as close as possible to it. Resistive strain gauges have been attached onto the tips of surgical graspers [8-10] and subsequently provide for the measurement of the forces during grasping operations. Sterilization of the arrangement as well as appropriate protection and shielding of the sensors are two of the primary issues associated with the use of resistive strain gauges on instrument tips.

Optical fiber sensors are being used as strain and force sensors in a number of areas including robotic surgery. Moreover, this mode of sensing offers a number of advantages, including, compact dimensions, immunity from electromagnetic interference and multiplexing capabilities.

The NeuroArm neurosurgical robotic system which is designed to be MRI compatible and can be used within a closed magnet bore [11] utilizes optical sensing methods. A three DOF optical force measurement scheme provides a sense of haptic perception to the user. It is unclear from the system description whether or not it has the capacity for measurement of grasping and scissor-cutting forces.

Park et al are investigating the feasibility of using FBG sensors in detecting the deflection of surgical needles in an MRI environment [12]. Results indicate that the FBG sensor can be used to estimate the tip deflection, but have also exhibited some hysteresis due to ineffective bonding at the sensor-needle interface.

Work carried out by Greenish et al employed "U" shaped strain gauge sensors (Entran ESU-025-1000), in the investigation of the forces generated along a pair of scissor blades, whilst cutting a range of anatomical tissues [13]. Forces on the scissor blades were found to be in the range of $2 \mathrm{~N}$ to $30 \mathrm{~N}$ for a range of biological tissue types. An evaluation testbed developed by Callaghan et al [14] facilitates the measurement of blade forces while cutting a range of synthetic tissue samples. Force values are in the range $2 \mathrm{~N}$ to $14 \mathrm{~N}$ with maximum blade strain of $121 \mu \varepsilon$.

This work presents preliminary investigations into the use of FBG's as force measurement sensors for robotic surgical end-effectors. An investigation was carried out to ascertain the minimum bond length required to ensure uniform strain over the FBG sensing element. Experimental validation of the FE results was carried out on a novel application-specific test rig incorporating a simplified geometrical realization of an actual surgical scissor blade. The rig design allows for the 
bonding of both an electrical strain gauge (ESG) and a $5 \mathrm{~mm}$ FBG sensor, enabling their respective performances to be evaluated for loads up to $30 \mathrm{~N}$. This loading induces strain values of up to $350 \mu \varepsilon$ in the blade allowing the effectiveness of strain transfer from blade structure to the FBG sensor to be evaluated.

\section{FBG Working Principle}

An elementary fiber Bragg grating comprises of a short section of single-mode optical fiber in which the core refractive index is modulated periodically using an intense optical interference pattern [15], typically at UV wavelengths. This periodic index modulated structure enables the light to be coupled from the forward propagating core mode into backward propagating core mode generating a reflection response. The light reflected by periodic variations of the refractive index of the Bragg grating, having a central wavelength $\lambda_{\mathrm{G}}$, is given by [16],

$$
\lambda_{G}=2 n_{e f f} \Lambda
$$

where $n_{\text {eff }}$ is the effective refractive index of the core and $\Lambda$ is the periodicity of the refractive index modulation.

The basic principle of operation of any FBG based sensor system is to monitor the shift in the reflected wavelength due to changes in measurands such as strain and temperature. The wavelength shift, $\Delta \lambda_{s}$, for the measurement of applied uniform longitudinal strain, $\Delta \varepsilon$, is given as,

$$
\Delta \lambda_{S}=\lambda_{G}\left(1-\rho_{\alpha}\right) \Delta \varepsilon
$$

where $\rho_{\alpha}$ is the photo elastic coefficient of the fiber given by the formula,

$$
\rho_{\alpha}=\frac{n^{2}}{2}\left[\rho_{12}-v\left(\rho_{11}-\rho_{12}\right)\right]
$$

where $\rho_{11}$ and $\rho_{12}$ are the components of the fiber optic strain tensor and $v$ is the Poisson's ratio. For a silica core fiber the value of $\left(1-\rho_{a}\right)$ is usually 0.78 . Thus, by measuring the wavelength shift, using techniques such as those described in [17], changes in temperature or strain can be determined depending on which parameter the FBG sensor is being used to measure.

A single mode optical fiber (SMF28) with an acrylate coating, containing an FBG of peak reflected wavelength of $1550 \mathrm{~nm}$ and a peak reflectivity $<70 \%$, was used in this experimental work. A $15 \mathrm{~mm}$ portion of the coating is removed to allow the $5 \mathrm{~mm}$ FBG to be written into the fiber core. A polyimide recoat, with a stiffness value greater than that of the acrylate, had then been applied over the 15 $\mathrm{mm}$ portion, providing for enhanced strain transfer. The shift in the FBG reflected 
wavelength due to strain is measured using an FBG interrogator from Smart Fibers Ltd. (Wx-02). The internal temperature sensor of the interrogator is used to compensate for ambient temperature fluctuations.

\subsection{Strain Transfer Theory}

Initial theoretical investigations into the strain transfer from a host material to a cylindrical fiber were carried out by Cox [18]. The resulting derived solution is adapted in this work to a four-layer cylindrical model for the purpose of identifying the strain transfer parameters which influence strain transfer between host material and fiber core. Following this, the derived expression is amended enabling the embedded model to be utilized for a surface mounted arrangement.

A bare fiber encapsulated within a protective coating, adhesive layer and the host material is illustrated in Fig. 1. The host material is the only element to which an axial load (z-direction) will be directly applied. The resulting strain will be transferred to the bare fiber as a result of shear strain within the two intermediate

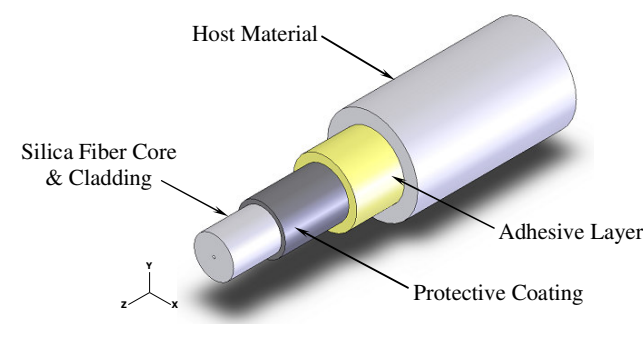

(a)

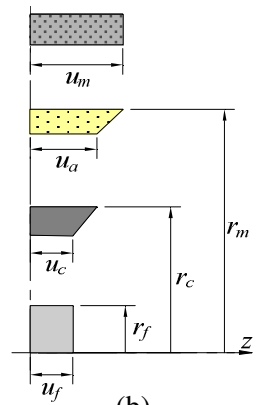

(b)

Fig. 1. (a) Structure of an optical fiber embedded within a host material (b) Material displacements and radii

layers. The average strain transfer coefficient (ASTC) $\bar{\alpha}$, is defined as the ratio of the average strain over the bonded fiber, $\bar{\varepsilon}_{f}$, to that of the host material, $\varepsilon_{m}$, and can be calculated using the following expression,

$$
\bar{\alpha}=\frac{\bar{\varepsilon}_{f}}{\varepsilon_{m}}=1-\frac{\sinh (k L)}{k L \cosh (k L)}
$$

where $L$ is the length of the FBG sensor and $k$ is the shear lag parameter encapsulating the material and geometric properties of the fiber, coating and adhesive layers and is given by, 


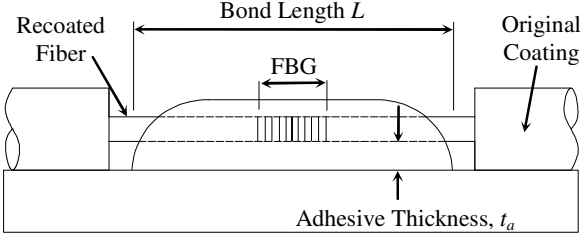

(a)

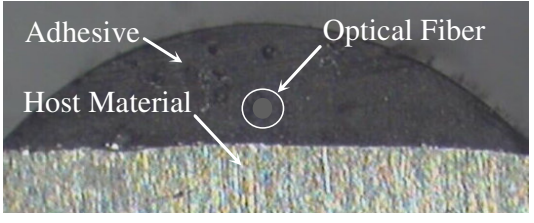

(b)

Fig. 2. (a) Surface bonded FBG sensor (b) View of the optical fiber within the adhesive layer

$$
k^{2}=\frac{2}{E_{f} r_{f}^{2}\left[\frac{1}{G_{c}} \ln \left(\frac{r_{c}}{r_{f}}\right)+\frac{1}{G_{a}} \ln \left(\frac{r_{m}}{r_{c}}\right)\right]}
$$

where $G_{c}$ and $G_{a}$ are the shear modulii values for the protective coating and adhesive layer respectively and $E_{f}$ being the Young's modulus of the fiber material.

A surface mounted fiber differs from an embedded fiber in that the host material does not fully encapsulate the coated fiber (Fig. 2(a)). A correction term introduced by [13] which incorporates the adhesive layer thickness $t_{a}$ into expression (5) ensures its applicability to a surface mounted fiber.

\subsection{FE Strain Transfer}

In practice the adhesive layer will take up a flattened profile (Fig. 2(b)) which is approximated as an ellipse for the purpose of this FE model. A previous study [19] demonstrated that variation in side width and top thickness of the elliptical profile had negligible effect on the strain transfer from host material to fiber core. It was shown, however, that the adhesive layer thickness, $t_{a}$, between the protective coating and the host material surface greatly influenced the average strain in the fiber core. FE models in this work are being used to study the effects of varying the adhesive layer thickness, bond length and stiffness values on the ASTC in a manner representative of a surface mounted FBG.

A $0.03 \%$ uniform strain was applied to the host material in the axial (z) direction for each simulation. The following parameters were varied and the strain distribution along the fiber core monitored after each simulation,

- The thickness of the adhesive layer between fiber coating and host material was varied from $10 \mu \mathrm{m}$ to $200 \mu \mathrm{m}$.

- The Young's modulus of the adhesive was set at either $2 \mathrm{GPa}$ or $3 \mathrm{GPa}$. 
The strain distribution along the fiber core for an adhesive Young's modulus of 3 GPa over a range of thicknesses from $10 \mu \mathrm{m}$ to $200 \mu \mathrm{m}$ is shown in Fig. 3(a). Trends in the data show that the strain distribution over the bonded $5 \mathrm{~mm}$ length is

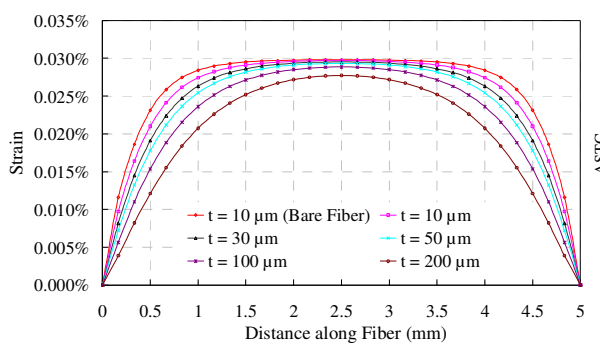

(a)

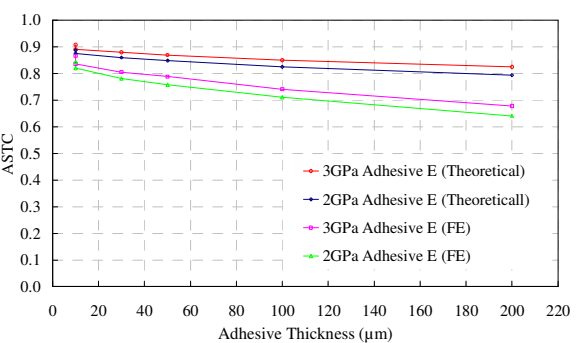

(b)

Fig. 3. (a) FE strain distribution along fiber core (b) FE and theoretical ASTC values for 5mm bond length

uniform over a region of approximately $1.5 \mathrm{~mm}$ for adhesive layer thicknesses between $10 \mu \mathrm{m}$ and $30 \mu \mathrm{m}$. A further reduction in the uniform strain length is observed for layer thicknesses between $50 \mu \mathrm{m}$ and $200 \mu \mathrm{m}$. The average value obtained from each of the hyperbolic strain curves in Fig. 3(a) represents the strain in the fibre core. Shear concentrations through both the adhesive and protective coating layers indicate that $100 \%$ uniformity in strain over the FBG length is unattainable by bonding the $5 \mathrm{~mm}$ FBG length only. The ratio of the average core strain to that of the host material (ASTC) is plotted in Fig. 3(b) for five different adhesive layer thicknesses and Young's modulus values of 2 and $3 \mathrm{GPa}$ over a $5 \mathrm{~mm}$ bond length only. Comparing the FE and the theoretical ASTC values it can be seen that strain transfer is most effective when the adhesive layer thickness is smallest $(10 \mu \mathrm{m})$ and its Young's modulus is greatest $(3 \mathrm{GPa})$. Reducing the stiffness of the adhesive from $3 \mathrm{GPa}$ to $2 \mathrm{GPa}$ has the effect of reducing the ASTC by an average of $2 \%$.

\subsection{Influence of Fiber Bond Length}

It can be seen from (4) that the ASTC is influenced by the shear lag parameter, $k$, which incorporates material and geometrical properties including adhesive layer thickness. The fiber bond length, $L$, also influences the effectiveness with which the host material strain is transferred to the FBG core. Achieving accurate strain measurement necessitates that a uniform strain distribution is obtained along the 5 $\mathrm{mm}$ grating region. This ensures that,

1. The strain sensitivity of the FBG $(1.2 \mathrm{pm} / \mu \varepsilon)$ is valid, as this value is based on the assumption of strain uniformity. 
2. No spectral broadening or distortion of the reflected wavelength spectra occurs which can result in measurement errors.

FE analysis was carried out to establish the minimum FBG bond length which ensures strain uniformity over the $5 \mathrm{~mm}$ grating. Five models were created using properties taken from an FBG employed in our experimental investigations, with the bond length being the only variable. Ideally, the adhesive layer thickness should be maintained as small as possible as outlined in Section 2.2, however achieving values in this range is prohibited by the technique employed for attachment of the fiber to the blade surface. This technique utilizes the dimensions of the original Acrylate coating $(250 \mu \mathrm{m})$ either side of the recoated portion $(133 \mu \mathrm{m})$, to maintain a consistent bond thickness of $58.5 \mu \mathrm{m}$ along the bond length. Results from the simulations showed that for an adhesive layer thickness of $58.5 \mu \mathrm{m}$, and a $4 \mu \mathrm{m}$ thick polyimide coating, a minimum bond length of $11 \mathrm{~mm}$ ensures uniform strain distribution along the $5 \mathrm{~mm}$ FBG (Fig. 4).

Fig. 4. FE strain distribution for various adhesive bond lengths at a fixed adhesive thickness of $58.5 \mu \mathrm{m}$

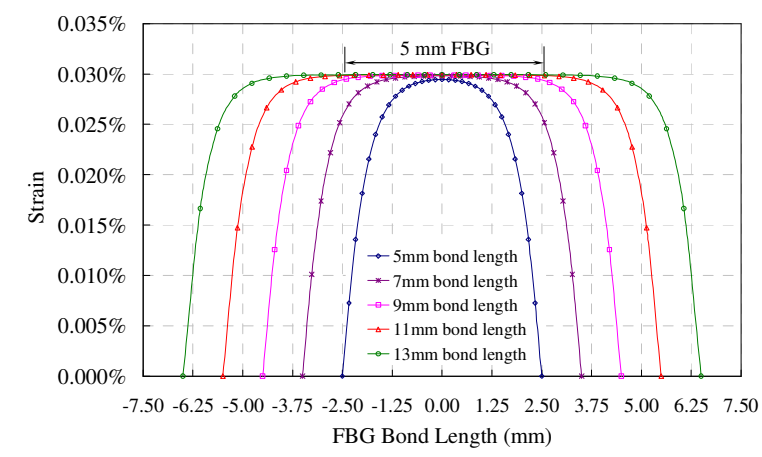

\subsection{Sensor Placement}

Strain measurements from the FBG sensor can be compared with those obtained from the FE model provided the strain field being measured by the sensor is uniform along the bonded grating length. Assuming that the scissor blade can be approximated as a uniformly tapered cantilever beam the point of maximum strain as well as the strain distribution over the bonded region of the fiber is established using the following expression,

$$
\varepsilon_{z}=\frac{6 F(L-z)}{E b(W-2 z \tan \phi)^{2}}
$$


where $z$ is the distance from the blade pivot point, $F$ is the applied load, $W$ is the thickness of the blade at its pivot, $b$ is the width of the blade, $E$ is the Young's modulus of the blade material and $\phi$ is half the angle of the blade (Fig. 5(a)).

The maximum strain was found to occur at a location $14 \mathrm{~mm}$ from the blade pivot point (Fig. 5(b)). Minimal strain variation (0.003\%) is estimated between $11.5 \mathrm{~mm}$ and $16.5 \mathrm{~mm}$ on the blade surface onto which the FBG sensor is attached. This small strain field variation will have negligible adverse effect on the reflected FBG signal.

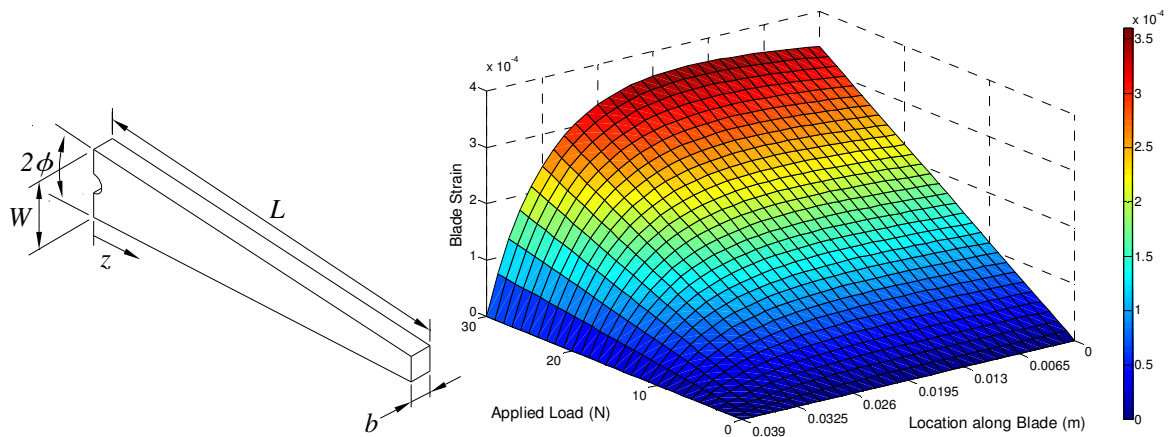

(a)

(b)

Fig. 5. (a) Blade geometry (b) Strain distribution on blade upper surface over a range of applied loads

Preliminary investigations into the nature of the strain values for blades of proportionately smaller dimensions revealed sufficiently large deflection and strain values still exist. Analysis of a small blade with $L=5 \mathrm{~mm}, b=1.5 \mathrm{~mm}$ and $W=2$ $\mathrm{mm}$ reveals that for loads up to $10 \mathrm{~N}$, maximum strain values of up to $270 \mu \mathrm{m}$ are achieved.

\section{Experimental Validation}

An experimental testing platform has been developed allowing investigation into the effects of adhesive layer thickness and bond length on the transfer of strain from a host material to an FBG fiber. The test rig consists of a simplified blade arrangement which is representative of one blade of a scissor end effector (Fig. 6). Blade dimensions ( $L=39 \mathrm{~mm}, b=2.286$ and $W=7.1 \mathrm{~mm}$ ) are comparable to that of a pair of straight blade Metzenbaum-Nelson scissors (nopa ${ }^{\circledR}$ instruments AC 110/18). The blade is symmetrical about its pivot point allowing for the simultaneous evaluation of an FBG strain sensor and an ESG. A self temperaturecompensated ESG (N11-MA-2-120) with a polyimide backing substrate was used as the reference strain sensor. This established strain measurement technology 


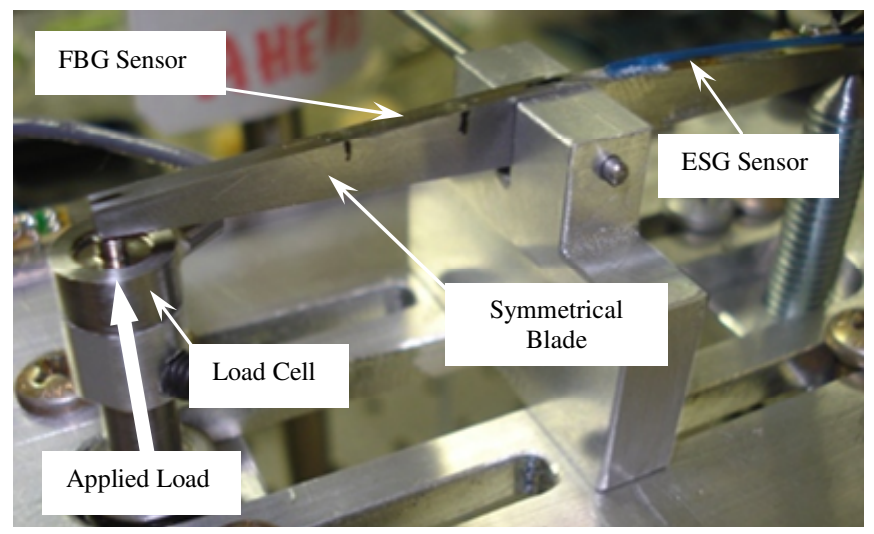

Fig. 6. Experimental test apparatus with surface mounted FBG and ESG strain sensors

serves as the best available sensing technique or performance comparison. These ESG readings represent the blade surface strain $\varepsilon_{m}$ at the fiber location, allowing an indication of the ASTC value (4) to be obtained.

Bonding of the FBG sensor to the surface of the blade was achieved using a special fixture incorporating four linear stages. This fixture allows the fiber to be fixed at one location while the blade is aligned in the $x, y$ and $z$ planes prior to the addition of the adhesive layer. After satisfactory alignment, the blade is lowered and the adhesive layer is manually applied over the bond length $(11 \mathrm{~mm})$. The blade is then raised to its original position where it meets the fiber which is held in position by an applicator containing a $250 \mu \mathrm{m}$ deep groove. This groove ensures that the fiber is fixed securely to the blade without squeezing the Acrylate coating at the points of contact between applicator and blade (either side of the recoated fiber). This technique is important since squeezing of the Acrylate coating during bonding results in curvature of the recoated fiber and consequently an inconsistent adhesive thickness along the adhesive bond length.

A range of loads in increments of $2 \mathrm{~N}$ between 0 and $30 \mathrm{~N}$ were applied to one end of the symmetrical blade resulting in an equal load being applied at the opposite end. This technique induces equal strain fields in each side of the blade. The reflected spectra of the FBG sensor were measured using an optical spectrum analyzer (Agilent 86140B). The measured spectra for zero strain and maximum applied load have the same bandwidth with only a peak shift due to the induced strain being observed (Fig. 7). This confirms that uniform strain is being induced over the grating length, as an appropriate bond length of $11 \mathrm{~mm}$ is being used.

The experimental data from the FBG and the ESG over the loading range of the blades are presented in Fig. 8(a). Blade strains obtained using FE and elementary beam theory are also presented. The strain values from the FBG sensor are in good agreement with those from the surface mounted ESG with a maximum of $2.8 \%$ variation in strain being observed over the full range. 
Fig. 7. FBG spectrum at zero load and maximum load

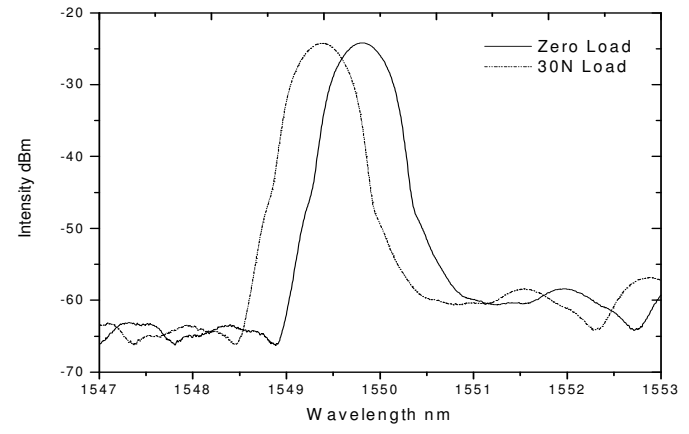

The theoretical ASTC is estimated using expression (4) where $\bar{\varepsilon}_{f}$ is the uniform strain within the fiber core and $\varepsilon_{m}$ is the uniform strain on the blade surface measured by the surface mounted ESG. The FE simulation indicates that an ASTC of 1 is obtainable using an appropriate bond length, adhesive layer thickness and coating thickness. An ASTC value of 0.98 is calculated using FBG and ESG strain values (Fig. 8(b)). This indicates the sensitivity of the FBG is comparable to that of the more established ESG technology signifying its suitability for the proposed measurement application. It is observed that the strain values from the FBG sensor

Fig. 8. (a) Experimental and theoretical strain (b) FBG and strain gauge comparison

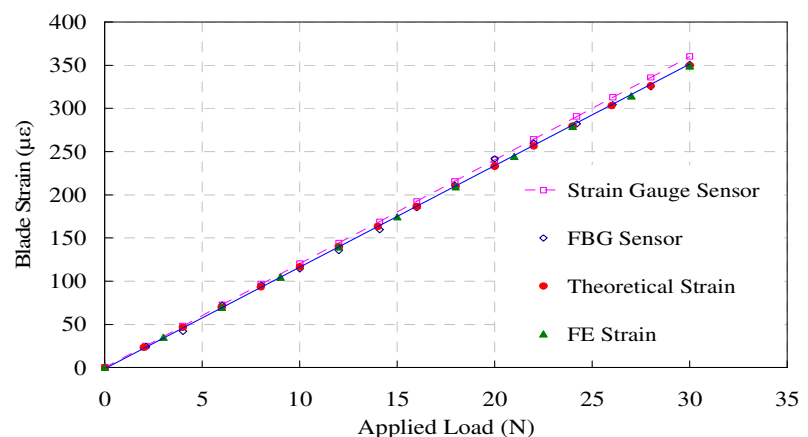

(a)

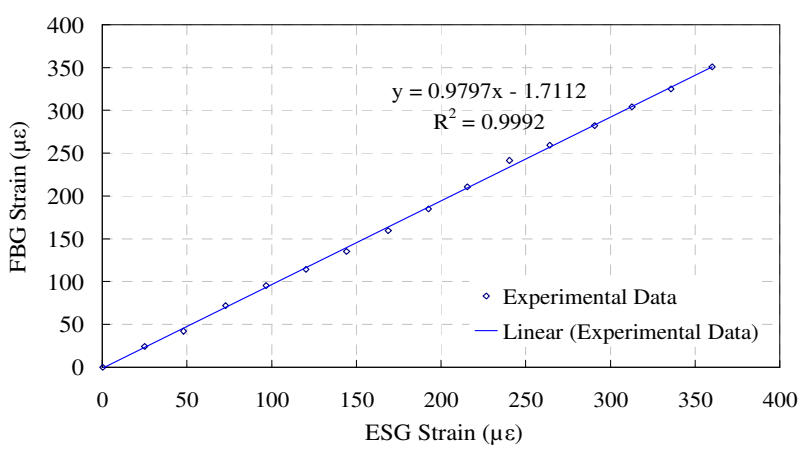

(b) 
correlate closely with both the analytical and $\mathrm{FE}$ values also indicating that a high level of strain transfer from blade to FBG is attained.

\section{Conclusions}

The aim of the present study is to assess the factors influencing strain transfer between the structure of a simplified scissor blade and the core of an FBG strain sensor. FEA has been employed to improve on the theoretical analysis by creating a more representative model of a surface mounted FBG sensor. Results revealed that for a fixed bond length, a thin adhesive layer with a high Young's Modulus value allowed maximum strain transfer to be achieved along the middle portion of the fiber only. It is essential that the fiber experiences a uniform strain over its full sensing length so that accurate strain readings from an FBG can be obtained. Further FE analysis based on the properties of the fiber revealed that for an adhesive layer thickness of $58.5 \mu \mathrm{m}$, a minimum bond length of $11 \mathrm{~mm}$ ensures both uniform strain distribution and maximum strain transfer to the FBG core.

A novel evaluation testbed was used which allows FBG and ESG strain readings to be assessed simultaneously. Results show that the sensors are in good agreement with a maximum variation of $2.8 \%$ between respective strain readings. The ratio of the strain measured by the FBG to that of the ESG was found to be 0.98 . Analysis of the reflected FBG spectrum at zero and maximum load reveals that no errors occurred in the FBG strain measurements as a result of strain nonuniformity along the grating. This indicates that $11 \mathrm{~mm}$ is an appropriate bond length for the $5 \mathrm{~mm}$ FBG used, allowing strain uniformity and complete strain transfer to be obtained.

It has been shown that an FBG sensor can be used in the measurement of strain on a simplified scissor blade arrangement as effectively as an ESG sensor. Ensuring appropriate bonding layer thicknesses and short fiber bond lengths widens the potential application of FBG sensors in the field of telerobotic surgery where the associated end-effectors have compact dimensions. Ongoing work involves incorporating the strain sensor into the blade structure, the aim of which is to make the FBG an integral part of the sensorized end-effector unit. In conjunction with this, an appropriate temperature compensation technique is being implemented which will improve the accuracy of the strain/force measurement system.

\section{References}

1. Tholey G, Pillarisetti A and Desai, J P (2004) On-site three dimensional force sensing capability in a laparoscopic grasper. Ind. Robot 31: 509-518

2. Kuebler B et al (2005) Development of actuated and sensor integrated forceps for minimally invasive robotic surgery. Int. J Med Rob and Comp Ass Surg 1: 96-107 
3. Berkelman P J et al (2003) A Miniature Microsurgical Instrument Tip Force Sensor for Enhanced Force Feedback During Robot-Assisted Manipulation. IEEE Trans Rob Auto 19: 917922

4. Tavakoli M et al (2005) Haptic interaction in robot-assisted endoscopic surgery: a sensorized end-effector. Int J Med Rob Comp Ass Surg 1: 53-63

5. Mayer $\mathrm{H}$ et al (2006) A system for robotic heart surgery that learns to tie knots using recurrent neural networks. Int Conf Intell Rob Syst: 543-548

6. Prasad S K et al (2003) A modular 2-DOF force-sensing instrument for laparoscopic surgery In: Ellis R E and Peters T M (eds.) MICCAI 2003, LNCS 2878, Springer Verlag, Heidelberg

7. Tholey, G., and Desai, J.P. (2007) A modular, automated laparoscopic grasper with threedimensional force measurement capability. Int Conf Rob Autom: 250-255

8. Fischer $\mathrm{G}$ et al (2006) Ischemia and Force Sensing Surgical Instruments for Augmenting Available Surgeon Information. Proc BioRob: 1030-1035

9. Wagner C R and Howe R D (2007) Force feedback benefit depends on experience in multiple degree of freedom robotic surgery task. IEEE Trans on Rob 23: 1235-1240

10. Dargahi J and Najarian S (2003) An endoscopic force-position sensor grasper with minimum sensors. Canadian J Elec Comp Eng 28: 155-161

11. Sutherland G R et al (2003) NeuroArm: an MR compatible robot for microsurgery. Int Congress Series 1256: 504-508

12. Park Y-L et al (2008) MRI-compatible Haptics: Feasibility of using optical fiber Bragg grating strain-sensors to detect deflection of needles in an MRI environment. Int Soc for Magnetic Resonance in Med (ISMRM)

13. Greenish, S. (1998) Acquisition and Analysis of Cutting Forces of Surgical Instruments for Haptic Simulation. (Thesis) Dep of Elec and Comp Eng: McGill University

14. Callaghan D J, McGrath M M and Coyle E (2007) A Force Measurement Evaluation Tool for Telerobotic Cutting Applications: Development of an Effective Characterization Platform. Int J Math, Phys Eng Sci 1: 144-150

15. Hill K O and Meltz G (1997) Fiber Bragg grating technology fundamentals and overview. J Lightwave Tech 15: 1263-1276

16. Rao Y-J (1997) In-fibre Bragg grating sensors. Measurement Sci Tech 8: 355-375

17. Wang Q et al (2006) Low-cost Wavelength Measurement based on a Macrobending Singlemode Fiber. Optics Letters 31: 1785-1787

18. Cox H L (1952) The elasticity and strength of paper and other fibrous materials. British J App Phys 3: 72-79

19. Wan K T (2008) Investigation of the strain transfer for surface-attached optical fiber strain sensors. Smart Materials and Structures 17: 035-037 